\title{
Doctors on demand
}

\author{
Navjoyt Ladher clinical editor
}

The BMJ

Oliver Cromwell reportedly suffered from renal stones, which feature on our cover this week. According to his doctor, as recorded in The Death of Oliver Cromwell, his preferred coping mechanism was "to swill down several sorts of liquor, and then stir his body by some violent motion ... that by such agitation he might disburden his bladder." Medical management has come on a lot since then, as Monica Morgan and Margaret Pearle describe in their State of the Art clinical review (doi:10.1136/ bmj.i52).

A modern day Cromwell would have a plethora of medical services to turn to. In an editorial this week, Jessica Watson and colleagues describe the growth in private online healthcare companies offering email, telephone, and video consultations (doi:10.1136/bmj.i1076). These companies are able to meet demand for convenient and accessible appointments, but what are some of the trade offs?

One adverse consequence may be overprescribing. While GPs and hospitals in England are being offered incentives to reduce antibiotic prescribing and stem levels of antibiotic resistance (doi:10.1136/bmj.i1499) one private online healthcare company sells travel packs containing antibiotics for self diagnosed infections.

And might clinical staff leave the NHS to work for these private providers, further depleting an already beleaguered workforce? The BMJ hosted a discussion at the Nuffield Trust Health Policy
Summit, exploring the current NHS crisis. Participants were asked whether the UK's medical workforce was fit for purpose and what could be done to improve things. As Gareth Iacobucci reports (doi:10.1136/bmj.i1510), there are already concerns that low morale and the dehumanising of medicine will adversely affect recruitment and retention of doctors and nurses.

So how should NHS general practice respond to the proliferation of private companies? The prime minister has established a challenge fund to stimulate innovative thinking around access to primary care. Watson and colleagues urge caution. New models of healthcare delivery should be "evaluated and evidence based but also timely and relevant to the population," they say. Figuring out just what is timely and relevant is no easy feat, but asking the population, or at least a sample of it, seems like a good start. Several years ago, general practices were offered financial incentives to establish patient participation groups in order to get feedback. But, as Stephen Gillam and Jennifer Newbould outline in this week's Analysis (doi:10.1136/bmj. i673), despite millions of pounds of investment the impact of these groups is unclear. There are several avenues for patient feedback available in primary care but, in a linked commentary (doi:10.1136/bmj.i913), Angela Coulter describes a "mixed bag of poorly evaluated methods" that don't seem to be serving patients or their primary care teams. It may therefore be a while before we know whether having doctors on demand is a good idea. 